\title{
Virtual Humans Playing the Role of Patients in Self-medication Consultations: Perspectives of Undergraduate Pharmacy Students
}

Ana Paula Cláudio ${ }^{1}$, Maria Beatriz Carmo ${ }^{1}$, Mara Pereira Guerreiro ${ }^{3,4}$, Afonso Cavaco ${ }^{2}$, Vítor Pinto ${ }^{1}$, Ana Pinha ${ }^{1}$

${ }^{1}$ BiolSI- Biosystems \& Integrative Sciences Institute, Faculty of Sciences, University of Lisboa, Lisboa, Portugal ${ }^{2}$ Faculty of Pharmacy, University of Lisboa, Lisboa, Portugal

${ }^{3}$ CiiEM-Instituto Superior de Ciências da Saúde Egas Moniz, Monte da Caparica, Portugal

${ }^{4}$ Escola Superior de Enfermagem de Lisboa, Lisboa, Portugal

\{apc, bc\}@di.fc.ul.pt, mguerreiro@egasmoniz.edu.pt, acavaco@ff.ulisboa.pt, \{fc41036, fc37397\}@alunos.fc.ul.pt ABSTRACT

i) an interactive application conceived to improve non-prescription medicines consultation skills in undergraduate Pharmacy students and

ii) a user test carried out with two sets of participants.

The Application
Virtual humans play the
role of patients and
communicate with the
student by speech and
by facial and body
language.
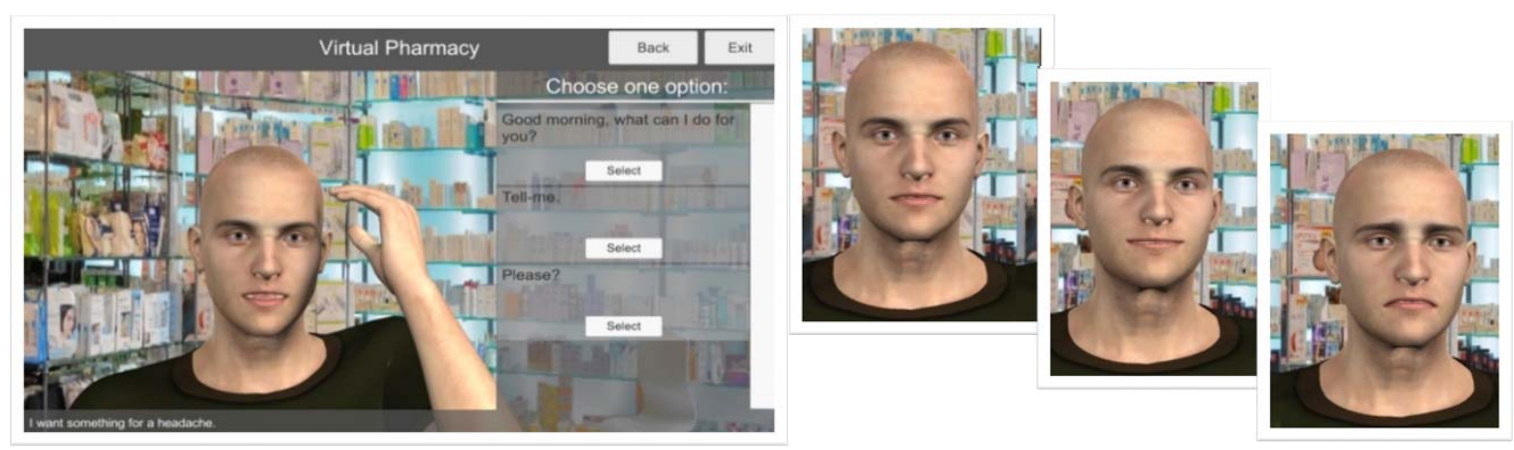

The application has two usage modes: the training mode, used autonomously by the student, and the assessment mode which is used by the teacher to evaluate the student's performance.

The Back Office and the Graph

\section{t.}

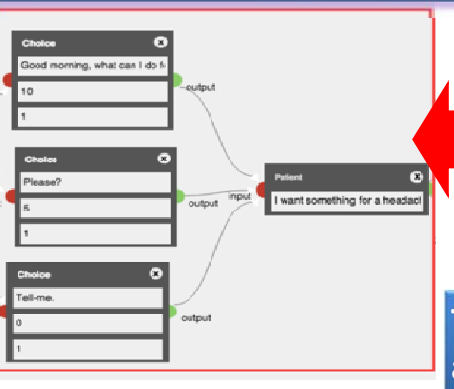

A BackOffice Web application was also implemented to assist teachers' work. It supports the collection of data about students' performance, the creation of new self-medication situations and their posterior insertion

$$
\text { in the application. }
$$

The teachers depict a graph of the dialogues for the new self-medication situation in an intuitive and user-friendly graphical interface.

The User Study

Two sets of Pharmaceutical Sciences students participated:

- 10 undergraduate students in the 4 th year ;

42 students performing their curricular internship behind a counter in a community pharmacy.

The overall results are quite positive; lower scores are related to the realistic appearance of the VH playing the role of patients.

The internships considered the prototype could become a powerful tool to learn and to consolidate knowledge, as long as it offers more situations. They mentioned that it can be a tool for students, but also for professionals that need to review and update their professional capacities: it can help remembering concepts and principles learned in school but already forgotten. 